\title{
High Resolution Radio and IR Observations of AGB Stars
}

\author{
W. Cotton ${ }^{1}$, G. Perrin ${ }^{2}$ R. Millan-Gabet ${ }^{3}$, O. Delaa ${ }^{4}$, \\ and B. Mennesson ${ }^{5}$ \\ ${ }^{1}$ National Radio Astronomy Observatory, 520 Edgemont Rd., Charlottesville, VA 202903, USA \\ email: bcotton@nrao.edu \\ ${ }^{2}$ LESIA, Observatoire de Paris, CNRA, UPMC, Université Paris-Diderot, Paris Sciences \\ et Letters, 5 place Jules Janssen, 92195, Meudon, France \\ email: guy.perrin@obspm.fr \\ ${ }^{3}$ California Institute of Technology, NASA Exoplanet Science Institute, Pasadena, CA 91125, \\ USA \\ email: R.Millan-Gabet@caltech.edu \\ ${ }^{4}$ Laboratoire Lagrange, UMR 7293 UNS-CNRS-OCA, Boulevard de l'Observatoire, B.P. 4229 \\ F, 06304 NICE Cedex 4, France \\ email: omar.delaa@oca.eu \\ ${ }^{5}$ Jet Propulsion Laboratory, California Institute of Technology, 4800 Oak Grove Drive, \\ Pasadena, CA 91190, USA \\ email: bertrand.mennesson@jpl.nasa.gov
}

\begin{abstract}
Asymptotic Giant Branch Stars (AGB) are evolved, mass losing red giants with tenuous molecular envelopes which have been the subject of much recent study using infrared and radio interferometers. In oxygen rich stars, radio $\mathrm{SiO}$ masers form in the outer regions of the molecular envelopes and are powerful diagnostics of the extent of these envelopes. Spectroscopically resolved infrared interferometry helps constrain the extent of various species in the molecular layer. We made VLBA $7 \mathrm{~mm} \mathrm{SiO}$ maser, Keck Interferometer near IR and VLTI/MIDI mid IR high resolution observations of the stars U Ari, W Cnc, RX Tau, RT Aql, S Ser and V Mon. This paper presents evidence that the $\mathrm{SiO}$ is depleted from the gas phase and speculate that it is frozen onto $\mathrm{Al}_{2} \mathrm{O}_{3}$ grains and that radiation pressure on these grains help drive the outflow.
\end{abstract}

Keywords. masers, stars: AGB and post-AGB, stars: imaging, stars: winds, outflows

\section{Introduction}

Asymptotic Giant Branch Stars (AGB) are low to intermediate mass stars that have exhausted their nuclear fuel, have become pulsating red giants and are losing most of their mass to become planetary nebulae. The extended, cool envelopes of these stars contain a variety of molecules some of which eventually condense into dust grains. In oxygen rich AGB stars, $\mathrm{SiO}$ masers form in the outer parts of the molecular envelope interior to where the silicate dust forms; see Reid \& Menton (1997), Danchi et al. (1994). Observations by Perrin et al. (2004) and Wittkowski et al. (2008) have shown that molecules in the envelope occur in shells.

Open questions about AGB stars are how do they sometimes form very asymmetric planetary nebulae and how is the mass loss driven. The observed silicate dust forms too far out in the envelope for radiation pressure on this dust to help drive the outflow. Recent speculation has centered on the role of $\mathrm{Al}_{2} \mathrm{O}_{3}$ dust which can form at a relatively high temperature $(\sim 1700 \mathrm{~K})$. Wittkowski et al. (2007) presented SiO maser and mid-IR 
Table 1. Primary IR Opacity Sources

\begin{tabular}{|l|c|}
\hline wavelength & Opacity sources \\
\hline near-IR & photosphere, inner molecular region \\
$7.80-9.30 \mu \mathrm{m}$ & SiO gas \\
$9.37-11.50 \mu \mathrm{m}$ & Silicate dust \\
$11.55-13.26 \mu \mathrm{m}$ & H2O gas \\
\hline
\end{tabular}
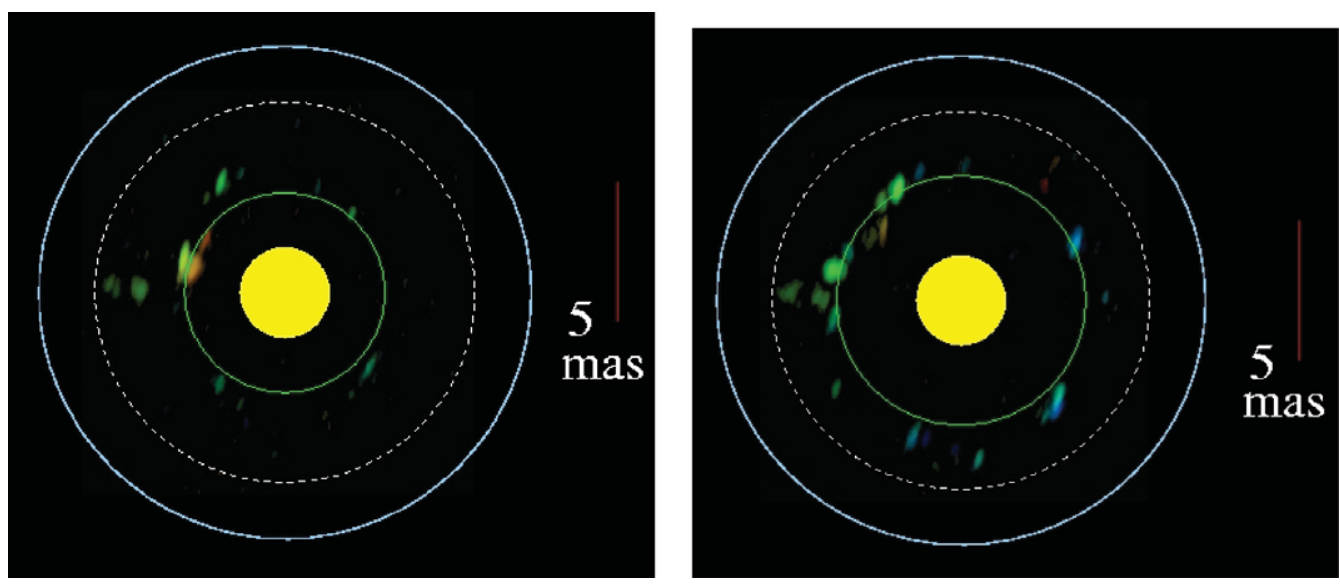

Figure 1. S Ser. On the left is the image in the $\mathrm{SiO} \nu=2, \mathrm{~J}=1-0$ transition. The green circle gives the fitted diameter on the maser ring, the light blue line the fitted size of the IR molecular shell and the dashed white line, the radius at which $\mathrm{Al}_{2} \mathrm{O}_{3}$ could condense. On the right is the image in the $\mathrm{SiO} \nu=1, \mathrm{~J}=1-0$ transition.

interferometric observations of the AGB star S Ori which they interpreted as showing $\mathrm{Al}_{2} \mathrm{O}_{3}$ dust forming just interior to the $\mathrm{SiO}$ masers.

\section{Observations}

A sample of AGB stars was observed using the VLBA, VLTI/MIDI and the Keck Interferometer. Multiple snapshot observations of the $7 \mathrm{~mm} \mathrm{SiO}$ masing $\nu=1, \mathrm{~J}=1-0$ and $\nu=2, \mathrm{~J}=1-0$ transitions near $43 \mathrm{GHz}$ were made on 1 July 2007 and 24 February 2008. Observations were analyzed as described in Cotton et al. (2006). The diameters of the masing regions were characterized by fitting circular rings.

Observations with the VLTI/MIDI (Leinert et al. 2003) measured between 7 and $14 \mu \mathrm{m}$; The Keck Interferometer observed S Ser at $2.2 \mu \mathrm{m}$. Models were fitted to the IR data to derive sizes of the photosphere and one or more layers needed to characterize the envelope seen in the mid IR. The mid-IR spectrum was modeled by wavelength ranges; the expected principle opacity sources of these are given in Table 1 .

\section{Results}

The observational results are given for S Ser in Fig. 1, W Cnc in Fig. 2, RX Tau in Fig. 3, U Ari in Fig. 4, V Mon in Fig. 5 and RT Aql in Fig. 6. For S Ser, only a single mid IR visibility was measured and a single layer model was fitted to the data. Too few maser spots were deteceted in U Ari and V Mon to reliably fit ring sizes. However, the minimum ring diameter for $\mathrm{V}$ Mon is the separation of the two spot groups which is very nearly the same as derived in the mid IR. No IR interferometric data were obtained 

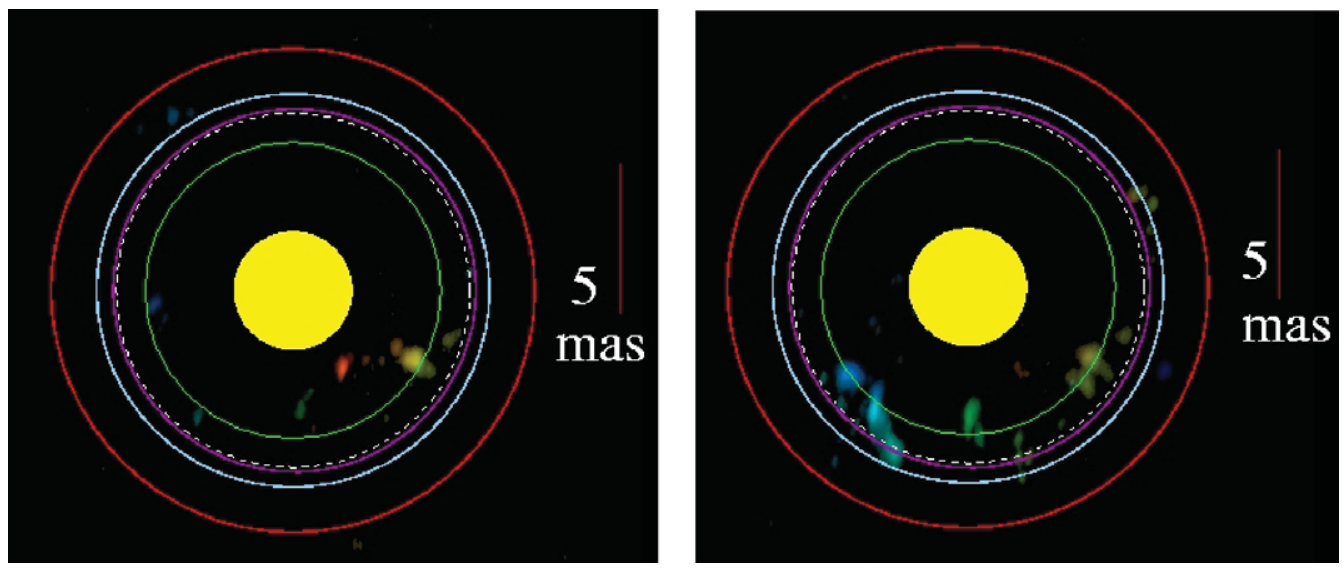

Figure 2. W Cnc. Like figure 1 except that the light blue circle shows the fitted size of the 7.8-9.3 $\mu \mathrm{m}$ data; magenta, the size of the $9.4-11.5 \mu \mathrm{m}$ and red, 11.6-13.3 $\mu \mathrm{m}$.
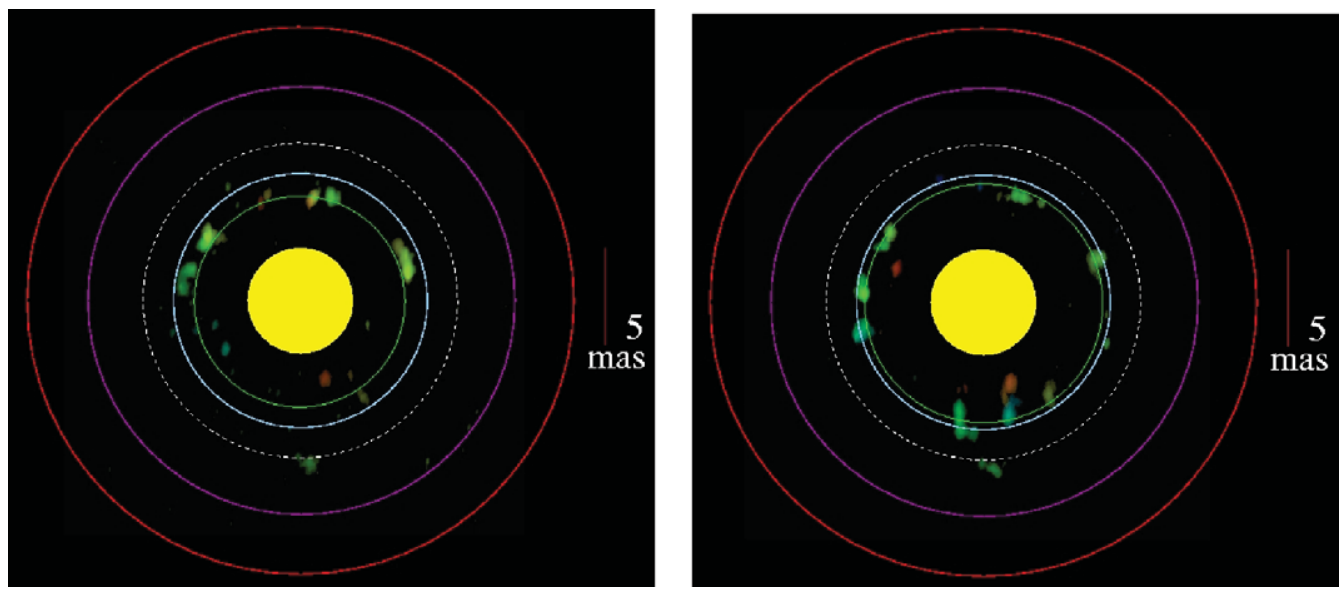

Figure 3. RX Tau. Like figure 2.
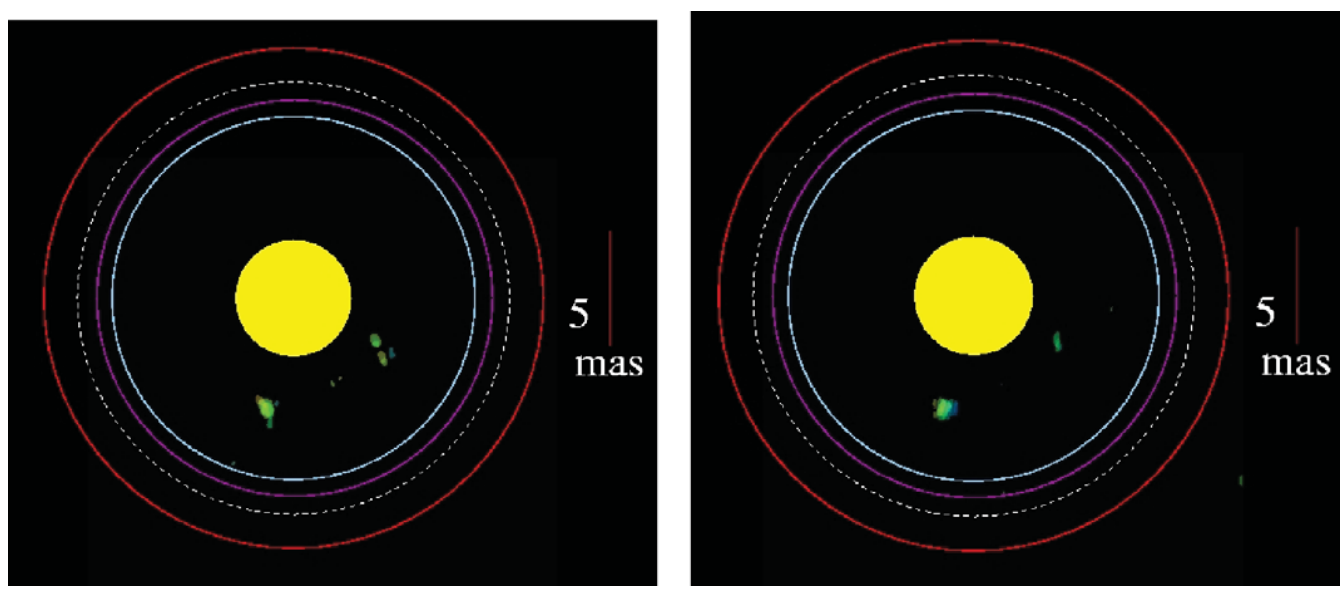

Figure 4. U Ari. Like figure 2 except that insufficient maser emission was present to fit a ring and align with the IR data. 


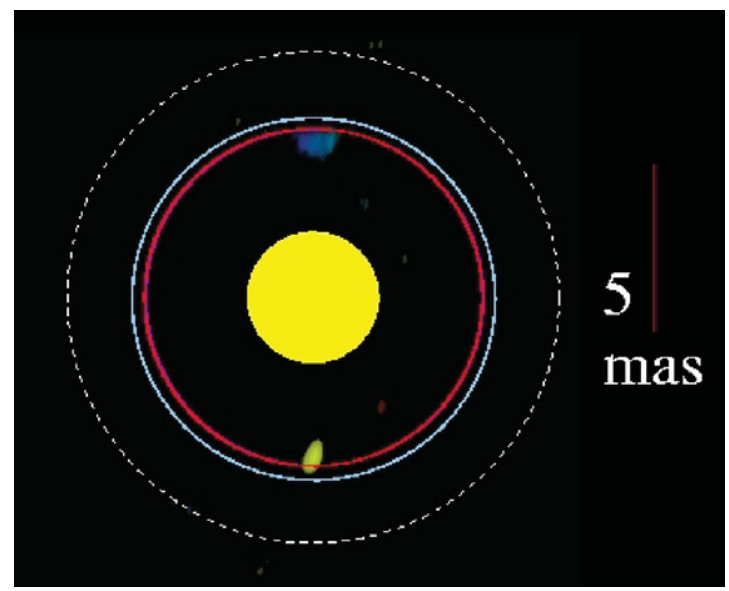

Figure 5. V Mon. Like figure 3 except that maser emission was only detected in the $\nu=1$, $\mathrm{J}=1-0$ transition.
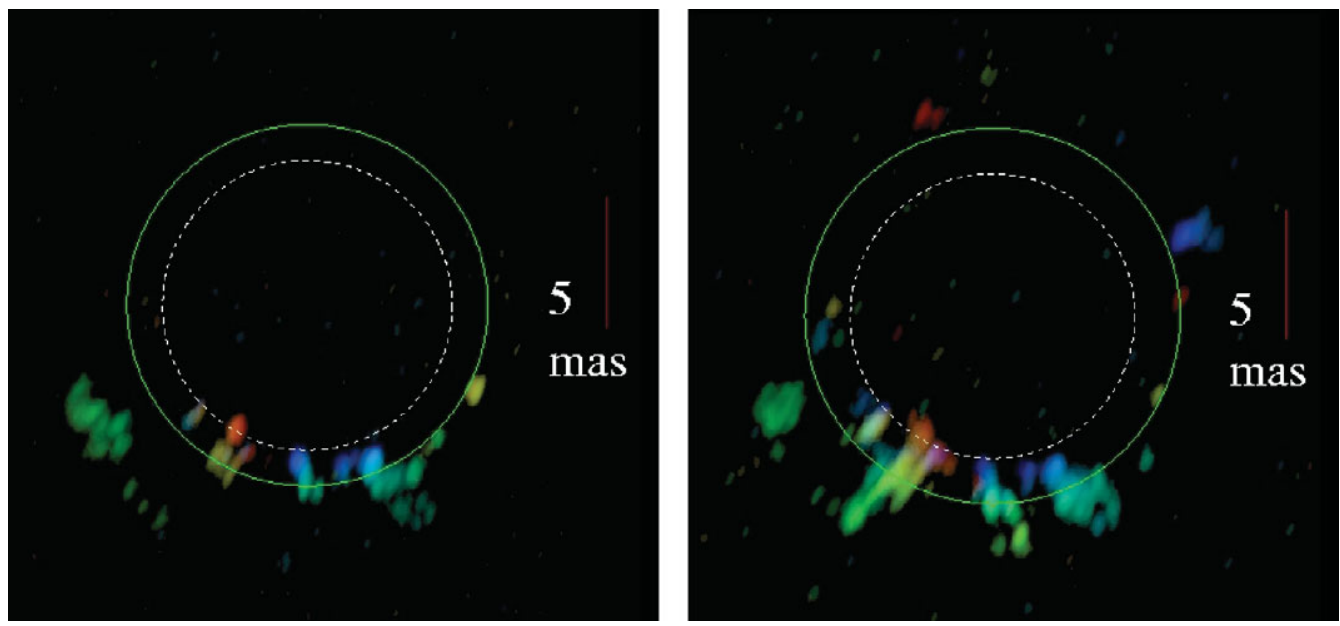

Figure 6. RT Aql. Like figure 2 except no IR interferometric data were obtained.

for RT Aql and the size of the photosphere, hence the anticipated $\mathrm{Al}_{2} \mathrm{O}_{3}$ condensation distance was based on photometric measurements from the literature.

\section{Discussion}

In none of the visibility spectra do we detect the strong $9.8 \mu \mathrm{m}$ silicate feature. Conversion of $\mathrm{SiO}$ to silicate must take place exterior to the region probed by this data.

There are two indicators of $\mathrm{SiO}$ gas in our data, the $\mathrm{SiO}$ masers and the $\mathrm{SiO}$ lines in the $7.80-9.30 \mu \mathrm{m}$ spectral region. With the possible exception of RT Aql, none of the stars observed show evidence for either of these diagnostics significantly exterior to the radius at which $\mathrm{Al}_{2} \mathrm{O}_{3}$ is expected to form (dashed circles in Figures 1-6). There was no IR interferometric data obtained for RT Aql and the photospheric size and the radius of $\mathrm{Al}_{2} \mathrm{O}_{3}$ condensation were estimated from photometric measurements in the literature. The ratio of the $\mathrm{SiO}$ maser ring size to the photospheric size is quite different for RT Aql and the values near 2.0 found in well constrained cases. 
Following the suggestion of Verhoelst et al. (2006), we propose that the gas phase $\mathrm{SiO}$ is condensing onto $\mathrm{Al}_{2} \mathrm{O}_{3}$ grains soon after these grains form. If this is the case, then the chemical conversion from $\mathrm{SiO}$ to silicates must take place on the dust grains. The radiation pressure on these dust grains with $\mathrm{SiO}$ mantles relatively close to the photosphere could then help drive the outflow.

\section{References}

Cotton, W. D., Vlemmings, W., Mennesson, B., et al. 2006, A\& A, 456, 339

Perrin, G., Coude Du Foresto, V., Ridgway, S. T., et al. 2004, A\&A, 426, 279

Reid, M. J. \& Menton, K. M. 1997, ApJ, 476, 327

Danchi, W. C., Bester, M., Degaicomi, C. G., Greenhill, L. J., \& Townes, C. H. 1997, AJ, 107, 1469

Leinert, C., Graser, U, Richichi, A., et al. 2003, The Messenger, 112, 13

Verhoelst, T., Decin, L., van Malderen, R., et al. 2006, A\&A, 447, 311

Wittkowski, M., Boboltz, D. A., Ohnaka, K., Driebe, T., \& Scholz, M. 2007, A\&A A, 470, 191

Wittkowski, M., Boboltz, D. A., Driebe, T., et al. 2008, A\&̈A, 479, L21 